Cahiers $d u$ MONDE RUSSE

\section{Cahiers du monde russe}

Russie - Empire russe - Union soviétique et États indépendants

$46 / 4 \mid 2005$

L'invention d'une politique humanitaire

\title{
Sarah Davies, James Harris, eds., Stalin: A New History
}

Alain Blum

\section{OpenEdition}

\section{Journals}

Édition électronique

URL : https://journals.openedition.org/monderusse/6596

DOI : 10.4000/monderusse.6596

ISSN : $1777-5388$

\section{Éditeur}

Éditions de l'EHESS

\section{Édition imprimée}

Date de publication : 1 décembre 2005

Pagination : 900-903

ISBN : 2-7132-2057-2

ISSN : $1252-6576$

Référence électronique

Alain Blum, "Sarah Davies, James Harris, eds., Stalin: A New History », Cahiers du monde russe [En ligne], 46/4 | 2005, mis en ligne le 03 juillet 2009, consulté le 03 septembre 2022. URL : http:// journals.openedition.org/monderusse/6596; DOI : https://doi.org/10.4000/monderusse.6596

Ce document a été généré automatiquement le 3 septembre 2022

Tous droits réservés 


\title{
Sarah Davies, James Harris, eds., Stalin: A New History
}

\author{
Alain Blum
}

\section{RÉFÉRENCE}

Sarah DAVIES, James HARRIS, eds., Stalin: A New History. Cambridge : Cambridge University Press, 2005, 308 p.

1 Cet ouvrage, composé d'un ensemble de chapitres essentiellement thématiques, a pour but de faire le point sur le regard que l'on porte désormais sur Stalin, sur son personnage, son rôle, son action, après plusieurs années d'exploration d'archives nouvelles et de renouvellement de l'historiographie de l'URSS. Il traite tant des années de formation de Stalin (qui, le rappelle Sarah Davies, a déjà 50 ans en 1929, à l'aube de l'apogée de son pouvoir) que de divers aspects de son implication politique.

2 Dans un premier chapitre, Alfred J. Rieber revient sur la jeunesse de Stalin, sur les nombreuses déformations apportées à l'histoire de cette période, en particulier par Stalin lui-même, et sur les orientations prises très tôt dans sa manière de gouverner. Puis Jeremy Smith analyse son passage au commissariat aux Nationalités en montrant que celui-ci fut décisif dans son approche future de la question des nationalités, même si Stalin ne participa pas activement à la gestion de cette institution.

3 James Harris étudie ensuite les archives du Secrétariat général du parti pour les années 1920 et tend à démontrer que le pouvoir acquis par Stalin comme secrétaire général ne consista pas tant à nommer les cadres du parti - ces nominations échappant pour la plupart, vu leur nombre, à un véritable contrôle du Secrétariat ou de Stalin lui-même qu'à exercer un pouvoir direct sur les responsables locaux en leur transmettant l'information et en répondant à leurs craintes d'être mis en cause et de perdre leur pouvoir. Cette stratégie visant à étouffer toute démocratie interne au parti dans le choix des cadres fut le facteur central qui permit à Stalin d'obtenir leur soutien. 
Les articles de J. Arch Getty d'un côté, d'Oleg V. Khlevniuk de l'autre, se penchent sur le pouvoir personnel de Stalin, en analysant son implication dans les prises de décision et le rôle des organes collectifs, le Politbjuro en particulier. J. Arch Getty engage une réflexion comparative sur le fonctionnement du Politbjuro et celui de cabinets ministériels européens, il montre par ailleurs combien Stalin participait peu aux processus de décision. Ce qui n'aurait, selon lui, aucun lien avec le nombre très faible des réunions du Politbjuro durant les années 1930. L'auteur estime que le pouvoir réel n'avait que peu de rapport avec ses formes institutionnelles, l'institution n'étant qu'une coquille renfermant des réseaux personnels d'individus qui, eux, prenaient véritablement les décisions. Cependant, la démonstration de Getty, stimulante, n'en est pas moins limitée dans la mesure où la nature même du pouvoir de Stalin n'est pas bien définie. Le fait qu'il ne s'implique pas dans $90 \%$ des décisions du Politbjuro n'apporte aucune précision sur la nature de son pouvoir, ni sur la menace constante d'intervention de sa part. De même, il parait difficile de nier complètement toute influence de l'institution. La contribution de Khlevniuk nuance d'ailleurs celle de Getty: Stalin - arbitre des tensions entre institutions et Stalin - maître de la répression fournissent deux clés pour comprendre en quoi consiste l'exercice de son pouvoir. S'il n'intervient pas dans de multiples cas, il est prêt à le faire à tout moment sur n'importe quel dossier, et son intervention est alors décisive: toute décision stalinienne est empreinte de violence politique. Mais, parallèlement, Khlevniuk tend à démontrer l'existence d'une autonomie bureaucratique conduisant au développement de relations de clientélisme, et ce $a$ fortiori à partir de 1950, lorsque Stalin s'écarte de plus en plus du pouvoir.

R. W. Davies restreint la problématique posée par les deux auteurs précédents à la prise de décision dans le domaine de la politique économique, et montre l'extrême intérêt porté par Stalin aux récoltes de grains, au détriment de l'agriculture dans son ensemble et même de l'industrie. Il montre qu'il pouvait manifester une certaine flexibilité, acceptant parfois, en particulier en 1932, de réduire les quantités à réquisitionner auprès des paysans, mais que par ailleurs il ne voyait jamais les conséquences dramatiques de ses décisions et s'obstinait souvent dans une voie menant au désastre.

Le dernier article de la première partie, dû à A. Rieber, traite de ce même sujet, mais appliqué à la politique extérieure, notamment au cours de la Seconde Guerre mondiale.

7 Vient ensuite une deuxième partie consacrée à la dimension idéologique du personnage. Elle est examinée tout d'abord dans deux contributions qui empruntent des voies différentes. D'un côté, Erik van Ree cherche à montrer que les fondements du mode de pensée chez Stalin, souvent considéré comme une sorte de russification du marxisme, n'ont rien de réellement hétérodoxe et sont déjà présents chez Marx lui-même ou chez ses émules. De l'autre, David Priestland, dans un chapitre particulièrement intéressant, examine comment Stalin agit et pense en alternant une vision scientiste et rationnelle, qui fait référence à des schèmes modernisateurs et technicistes, et une vision qu'il nomme "quasi romantique », privilégiant le volontarisme et l'action - le politique de manière plus générale - face à la technique.

8 La mobilisation par la propagande est l'une des préoccupations de Stalin, ce qui permet de comprendre l'intérêt considérable qu'il porte au cinéma et le soutien qu'il lui prodigue au milieu des années 1930, comme le montre Sarah Davies dans une étude qui analyse les notes de Boris Šumjackij (directeur de Sojuzkino entre 1930 et 1937), prises lors de projections privées en présence du vožd'. 
9 William Chase examine les procès publics des années 1936-1938 et le rôle qu'y joua Stalin personnellement en les présentant comme une réponse aux craintes de la population devant diverses menaces. Ce chapitre ne permet cependant pas de cerner la part de Stalin dans l'organisation de ces procès, comme le reconnaît l'auteur. L'histoire de l'élaboration des biographies rédigées du vivant de Stalin permet à David Brandenberger de souligner d'une part leur apparition tardive et, d'autre part, le rapport ambigu et changeant de Stalin à l'égard du culte de la personnalité - soit deux faits que l'auteur relie, de manière semble-t-il contradictoire.

10 Enfin Ethan Pollock revient sur les nombreuses interventions de Stalin dans le domaine scientifique en abordant deux exemples particulièrement intéressants : la disgrâce du linguiste Marr et l'incapacité de Stalin à achever de son vivant la rédaction d'un cours d'économie politique. Ses prises de position dans ce champ sont nombreuses et ont conduit, après guerre, à remettre en valeur une science auparavant désorientée par une idéologie marxiste interprétée au premier degré (comme, par exemple, l'évolution des langues, interprétée par la linguistique au travers de la dimension de classe), mais où l'idéologie reste présente, ou plutôt où l'on continue à établir un lien entre progrès scientifique et idéologie.

11 Ainsi, ce livre présente l'intérêt de fournir une série de " portraits », de schémas d'action ou de pensée, de montrer un Stalin aux multiples facettes. Si tous ces textes s'appuient sur des matériaux d'archives nouveaux, leur juxtaposition ne suffit cependant pas à donner une cohérence générale à l'ouvrage. Et on ne fait qu'approcher les modes d'autorité et de commandement de Stalin, ainsi que la mise en relation de son parcours propre avec le contexte social des années de genèse de son pouvoir. Sans doute est-ce la loi du genre, mais on aurait souhaité que les thèmes traités soient davantage élaborés, ce qui aurait sans doute permis d'obtenir in fine des développements conceptuels plus approfondis. Quoi qu'il en soit, la lecture reste passionnante, suscite maintes interrogations et témoigne du renouvellement profond du regard que l'on porte sur l'homme Stalin.

\title{
AUTEURS
}

\author{
ALAIN BLUM
}

